\title{
Titan's young surface: Initial impact crater survey by Cassini RADAR and model comparison
}

\author{
R. D. Lorenz, ${ }^{1}$ C. A. Wood, ${ }^{2}$ J. I. Lunine,${ }^{3}$ S. D. Wall, ${ }^{4}$ R. M. Lopes ${ }^{4}$ K. L. Mitchell, ${ }^{4}$ \\ F. Paganelli, ${ }^{4}$ Y. Z. Anderson, ${ }^{4}$ L. Wye,${ }^{5}$ C. Tsai, ${ }^{5}$ H. Zebker, ${ }^{5,6}$ and E. R. Stofan ${ }^{7}$ \\ Received 1 December 2006; revised 11 February 2007; accepted 7 March 2007; published 12 April 2007.
}

[1] Cassini's Radar instrument has mapped over $10 \%$ of Titan's surface through the T18 flyby in August 2006 and has detected remarkably few impact craters. Three impact craters have been conclusively identified and named, and a handful of unconfirmed candidate impact structures are documented. These results indicate that the overall crater density is a factor of $\sim 5$ higher than Earth, and has a sizedistribution slope very similar to Earth's indicating rapid obliteration of smaller structures by erosion and burial. The paucity of small craters was expected from atmospheric shielding considerations, but the rarity of medium (20$100 \mathrm{~km}$ diameter) craters - some 30-300 times less abundant than on nearby Saturnian satellites - implies rapid resurfacing. Erosion and burial appear to be the dominant means of crater obliteration, with viscous relaxation playing a lesser role. Models of the 20-100 km impact crater production rate suggest an overall crater retention age of $100 \mathrm{Myr}-1 \mathrm{Gyr}$, the same as the corresponding age for the Earth. Citation: Lorenz, R. D., et al. (2007), Titan's young surface: Initial impact crater survey by Cassini RADAR and model comparison, Geophys. Res. Lett., 34, L07204, doi:10.1029/ 2006GL028971.

\section{Introduction}

[2] The joint NASA-ESA-ASI Cassini-Huygens mission is presently 2 years into what is planned to be a 4 -, and likely 6- or more, -year comprehensive exploration of the Saturnian system. About 20 Titan flybys have been made, on 8 of which (through T18) synthetic aperture radar (SAR) mapping has been performed by Cassini's Radar instrument, revealing a strikingly varied landscape [Elachi et al., 2005, 2006]. Although only a little over $10 \%$ of Titan's surface has been covered to date (Figure 1), an initial report of Venus cratering from Magellan [Phillips et al., 1991] when that mission had attained about $15 \%$ coverage gave results that turned out to be representative of the planet as a whole

\footnotetext{
${ }^{1}$ Space Department, Applied Physics Laboratory, Johns Hopkins University, Laurel, Maryland, USA.

${ }^{2}$ Center for Education Technology, Wheeling Jesuit College, Wheeling, West Virginia, USA.

${ }^{3}$ Lunar and Planetary Laboratory, University of Arizona, Tucson, Arizona, USA.

${ }^{4}$ Jet Propulsion Laboratory, California Institute of Technology, Pasadena, California, USA.

${ }^{5}$ Department of Electrical Engineering, Stanford University, Stanford, California, USA.

${ }^{6}$ Department of Geophysics and Department of Electrical Engineering, Stanford University, Stanford, California, USA.

${ }^{7}$ Proxemy Research, Bowie, Maryland, USA.
}

Copyright 2007 by the American Geophysical Union. 0094-8276/07/2006GL028971\$05.00
(135 craters out of $\sim 1000$ ), so a Cassini progress report seems timely. This paper will focus on the abundance and size distribution of the observed craters-specific morphological investigations are discussed elsewhere (C. A. Wood et al., manuscript in preparation, 2007).

[3] The density of craters $20 \mathrm{~km}$ diameter or greater on other Saturnian satellites [Lorenz, 1997] is 100-200 craters of per million $\mathrm{km}^{2}$ (roughly the area covered in one full SAR pass, slightly more than $1 \%$ of Titan's surface.) The density of craters smaller than $20 \mathrm{~km}$ adds only modestly $(<\sim 50 \%)$ to the total on Titan, since its thick atmosphere would screen many of these impactors out [e.g., Engel et al., 1995; Ivanov et al., 1997; Korycansky and Zahnle, 2005].

[4] Crater studies on Titan pose challenges to optical remote sensing as the opacity of Titan's haze reduces the observable resolution and mutes shadowing effects. Thus while the Cassini ISS camera has substantial low-latitude and southern hemisphere coverage, only the larger and high albedo-contrast features can be observed. Several candidate impact structures were noted in early reconnaissance by ISS [Porco et al., 2005], including a large dark ring (seen in radar to be the $\sim 400 \mathrm{~km}$ crater Menrva) and several bright rings in dark terrain, including Guabonito - described below. The VIMS instrument on Cassini, operating at longer wavelengths of lower opacity is more sensitive to shadowing, but is not able to efficiently map large areas at high resolution. Thus the $\sim 40 \%$ of Titan flybys on which SAR observations were made to date form the basis of the crater density measurements reported here. As always, there is subjectivity in the identification of impact features which leads to an uncertainty of a factor of $\sim 2$ in their number, but the overall conclusions about the age of the surface are not affected.

\section{Radar Observations}

[5] The first radar pass (TA, October 2004) remarkably showed no impact structures at all [Elachi et al., 2005], even though coverage of the equivalent area on other Saturnian satellites would have detected hundreds. Some probable cryovolcanic features were noted, providing a possible explanation for the paucity of craters. In contrast, the T3 swath (February 2005 [Elachi et al., 2006]) showed two large structures, named Menrva (the outermost ring is $\sim 440 \mathrm{~km}$ diameter, although the nominal diameter is $392 \mathrm{~km}$ ) and Sinlap (80 km diameter, see Figure 2). This swath also showed fluvial channels, indicating another mechanism by which craters might be degraded. T7, in September 2005, sampled only half the planned area at high southern latitudes owing to a spacecraft problem, but did show one very small possible structure (Feature $\mathrm{H}$ in Figure $3, \sim 4 \mathrm{~km}$ in diameter), together with further evidence of fluvial processes. 


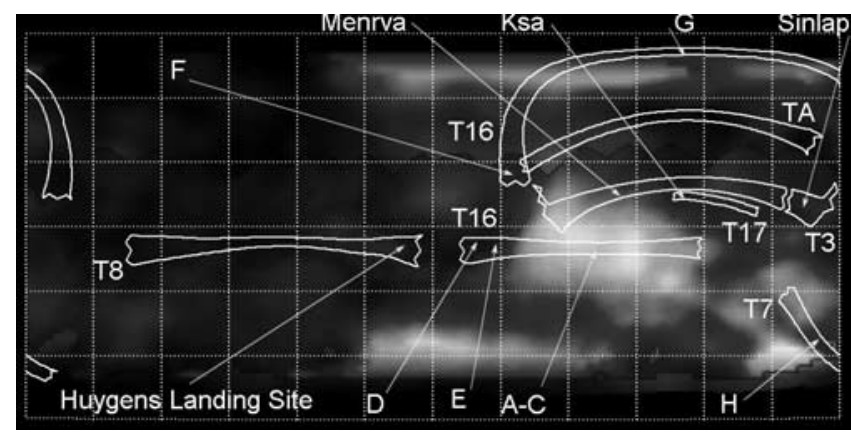

Figure 1. Location map, centered on the antiSaturn point of Titan - large bright region right of center is Xanadu. Map is $940 \mathrm{~nm}$ albedo from Cassini ISS and Hubble Space Telescope data. Grid boxes are 30 degrees across. SAR swaths are indicated and labeled, with features in Figures 1 and 2 arrowed. Most coverage, and essentially all the identified impact features, are on Titan's leading hemisphere, the right half of the map.

T8 in October 2005 revealed no obvious impact features but showed the large equatorial dark areas to be covered with massive fields of sand dunes, again indicating a mechanism for burial. Many small circular and near-circular features were detected in $\mathrm{T} 7$ and $\mathrm{T} 8$ but are believed to be cryovolcanic in origin (J. I. Lunine et al., Cassini Radar's third and fourth looks at Titan, submitted to Icarus, 2006).

[6] Flyby T13 in May 2006 covered the large bright area Xanadu, and revealed several structures that may be impact features. The radar-bright ring Guabonito (Feature E in Figure 3, $80 \mathrm{~km}$ across) was observed to have a similar appearance as it has optically: the higher radar resolution and contrast shows the dark center of the feature and the surrounds to be streaked with dark dunes trending to the southeast. Guabonito may be the bright remnant rim of an impact crater buried under the dunes, although a volcanic origin for this feature and several that follow is possible we defer detailed geological analysis to future work. An enigmatic near-circular, perhaps polygonal, feature (Feature D in Figure 3) appears in the optically-bright area Shikoku Facula. This feature, perhaps an impact crater with structural control of shape, as Meteor crater on Earth, is not apparent in optical images of the area [Porco et al., 2005].

[7] T13 also showed several possible relict impact structures (Features $\mathrm{A}-\mathrm{C}$ in Figure 3 ) in the highly rugged Xanadu terrain. An overall discussion of Xanadu is presented elsewhere (R. L. Kirk et al., manuscript in preparation, 2007). Feature A can be interpreted as the $\sim 50 \mathrm{~km}$ rim of a buried/flooded crater, like many on the Earth's moon. Feature B, fractionally larger, appears to show a central peak complex. Feature $\mathrm{C}$ is a somewhat flat-floored circular feature that could be plausibly interpreted as a crater. Several other structures not indicated are similar but less convincing.

[8] T16 in July 2006 showed another bright ring at the low-latitude part of the swath (Feature F in Figure 3 is as yet unnamed). It is a similar size to Guabonito, but with a bilateral symmetry possibly indicative of ejecta from an oblique impact. (A third indistinct but comparable-sized ring, not shown, was observed on T8, just to the east of the Huygens site.) The high-latitude part of T16 revealed lakes up to $\sim 100 \mathrm{~km}$ across: some of the smaller examples (5$10 \mathrm{~km}$ across) are within rather steep-sided circular depressions, and are surrounded by bright halos reminiscent of blocky ejecta fields (Feature $\mathrm{G}$ in Figure 3). These seem superficially reminiscent of crater fields from disrupted impactors [e.g., Korycansky and Zahnle, 2005], but detailed study and context [Stofan et al., 2007] shows cryovolcanism and karstic sapping to be more likely formation mechanisms.

[9] The T17 flyby in August 2006 showed a $29 \mathrm{~km}$ diameter crater named Ksa (in a short swath: radar was not the prime observer on this pass), not far from Menrva. The T18 swath, adjacent to T16, was also a short one and showed no impact structures.

[10] To summarize, in addition to the three named craters, there are about half a dozen other candidate structures with diameters large enough (some tens of $\mathrm{km}$ ) to be interpretably resolved. The resultant crater density, taking into account the size-dependent sampling area (see next section), is shown in Figure 4. The density is orders of magnitude smaller than that expected - a conclusion that is insensitive to whether none, or half, or all of the candidate structures above are determined to be impact in origin.

\section{Crater Population Survey}

[11] Although the radar imaging coverage of Titan is far from complete, even the data at hand usefully constrains the

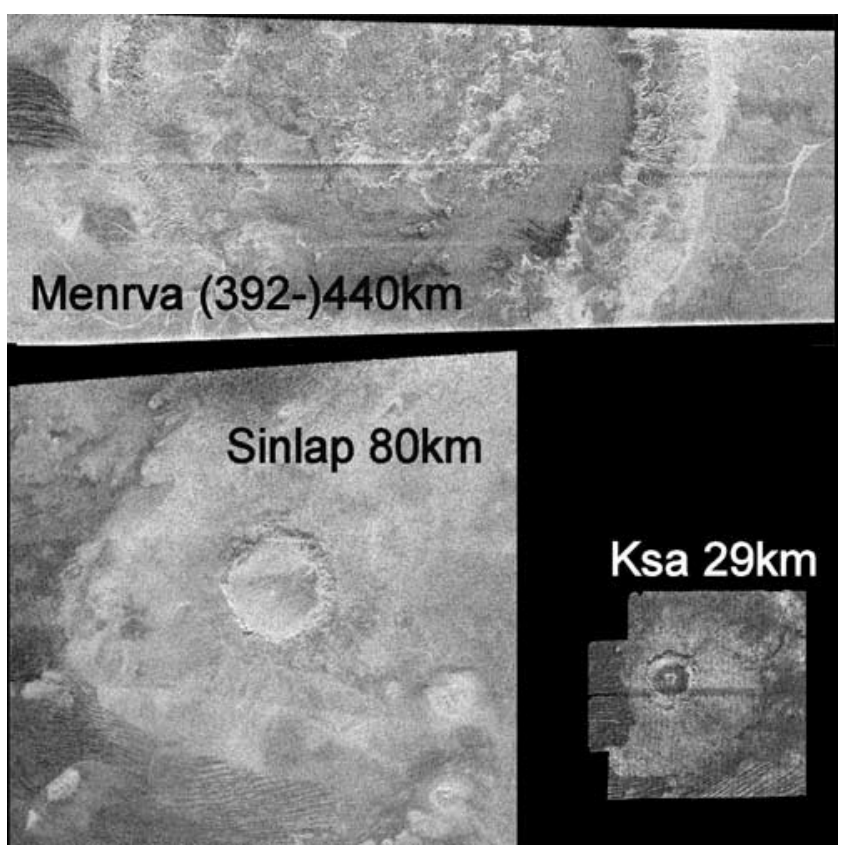

Figure 2. Montage at same scale (128 pixels/degree) of the three confidently-identified and named impact structures, (top) Menrva $\left[15.2-21.3^{\circ} \mathrm{N}, 82.3-92.3^{\circ} \mathrm{W}\right]$ at $\sim 400 \mathrm{~km}$, (bottom left) Sinlap $\left[10.2-12.3^{\circ} \mathrm{N}, 15.2-17^{\circ} \mathrm{W}\right]$ at $80 \mathrm{~km}$, and (bottom right) $\mathrm{Ksa}\left[13.4-14.5^{\circ} \mathrm{N}, 64.8-65.9^{\circ} \mathrm{W}\right]$ at $29 \mathrm{~km}$. North is up in each case - note the tendency of fluvial channels and Aeolian features to trend eastwards and the better-preserved Eastern rim of Menrva. Near-horizontal stripes across the whole images are radar imaging artifacts. Radar illumination is from above with incidence angles of $\sim 21^{\circ}, 14^{\circ}$ and $34^{\circ}$ respectively. 


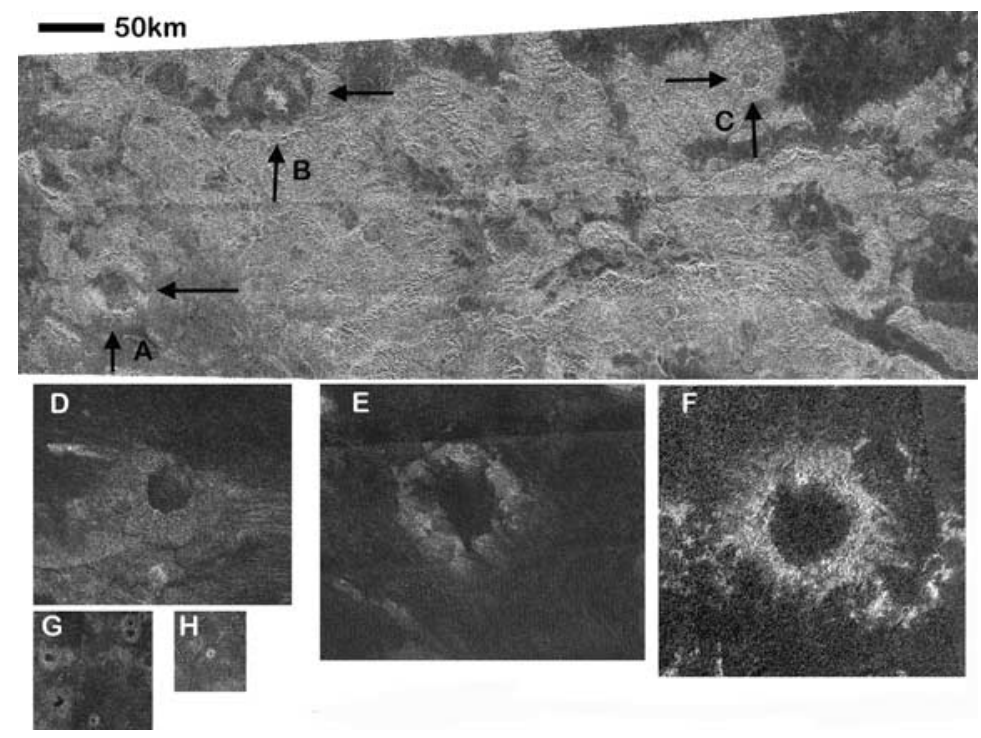

Figure 3. Possible impact features identified in radar data. Features $\mathrm{A}-\mathrm{E}$ were imaged on T13; F and $\mathrm{G}$ on $\mathrm{T} 16$ and $\mathrm{H}$ on T7. A-C are in Xanadu: Feature A appears to be a remnant rim of a buried crater; Feature B similarly appears flooded or buried, and the possible central peak complex is remarkable given then lack of same on the similar-sized Sinlap crater. Feature $\mathrm{C}$ is an example (others can be seen in the image) of structures that are possible but unlikely crater candidates. Feature $\mathrm{D}$ is in the northern part of Shikoku, and has an intriguing polygonal shape, indicating possible structural control by faulting: it is not apparent in optical images. Feature E (named Guabonito) may be a rim buried by dark sand dunes and appears essentially identical in optical data. A subset of dark lakes imaged at high northern latitudes (Feature G) are circular in shape, in some cases appear in clusters, and are surrounded by bright halos. Small Feature H is anomalous in that no similar features are seen elsewhere in that swath - although too small to resolve morphological detail it seems a reasonable candidate as an impact structure.

crater population. The long, thin images generated by SAR are in fact a rather efficient way of hunting for large craters (more so, per unit area covered, than a square image or mosaic). This is because only the rim, not the center, of the crater needs to be seen to know that the crater exists. For a swath of width $\mathrm{w}$ and length L, (typically $\sim 300 \mathrm{~km} \times$ $4000 \mathrm{~km}$ ) the area covered by the image is wL, but the effective sampling area for craters of diameter $\mathrm{D}$ approaches $\sim(\mathrm{w}+\mathrm{D})(\mathrm{L}+\mathrm{D})$. For large craters, the improvement is substantial - a factor of $\sim 2$ sampling area for $300 \mathrm{~km}$ craters.

[12] On a large plane of area A, the probability of detection with a swath is analytically $\sim(\mathrm{w}+\mathrm{D})(\mathrm{L}+\mathrm{D}) / \mathrm{A}$. However, the sampling efficiency is much stronger on the finite surface of a spherical planet covered by several widely-spaced swaths, since if a given large crater misses one swath, it is increasingly likely to be clipped by another. Thus, a simple Monte-Carlo experiment was conducted as follows. A $360 \times 180$ array was set with a flag for those latitude/longitudes covered by SAR to date. The center location for an impact crater of specified diameter (20$1000 \mathrm{~km}$ ) was chosen at random and spherical trigonometry was used to set elements in a separate $360 \times 180$ lat-lon array where the rim would be present. Where the product of the two arrays is non-zero, the crater would have been detected in the SAR imaging to date. (The $1^{\circ}$ or $\sim 2000 \mathrm{~km}^{2}$ overlap criterion is arbitrary, but reasonable.) The experiment was repeated 1000 times to determine the probability of detecting a crater so far.

[13] The experiment indicates the probability of detecting a $450 \mathrm{~km}$ crater within the area imaged so far as about $26 \%$, suggesting there may be $\sim 3$ more Menrva-sized structures

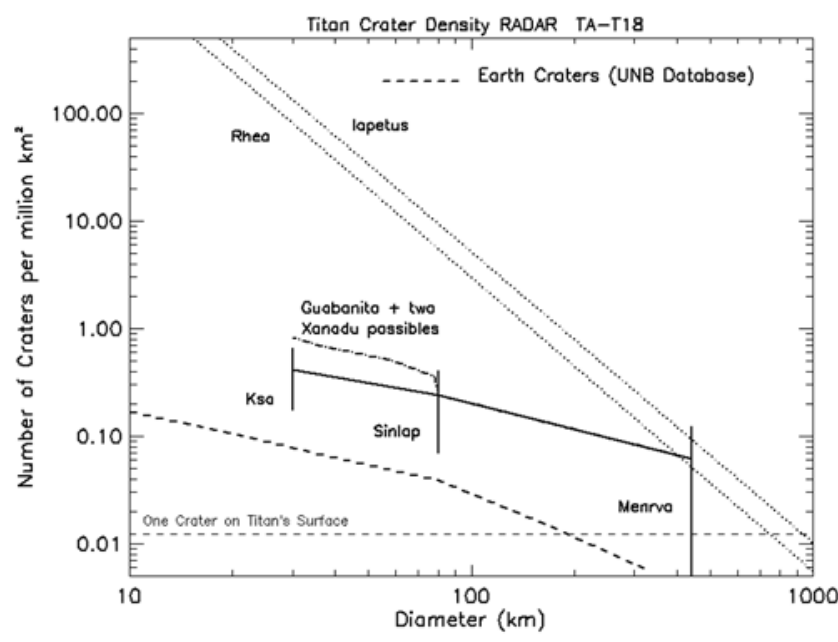

Figure 4. Cumulative impact Crater Population on Titan. The solid line is defined by the three known craters and the diameter-dependent survey area of the radar coverage to date. One-sigma error bars are shown. If $\sim$ half the candidate structures are interpreted as impact craters, the mediumcrater population rises slightly as indicated by the dash-dot line. In any case, the distribution for craters of $100 \mathrm{~km}$ or less is orders of magnitude smaller than for comparable Saturnian satellites (power law fits to Rhea and Iapetus populations are shown). The slope of the Titan crater distribution resembles that of the Earth (see the University of New Brunswick available at http://www.unb.ca/passc/ ImpactDatabase/), although with a number density of craters about $5 \mathrm{x}$ higher. 


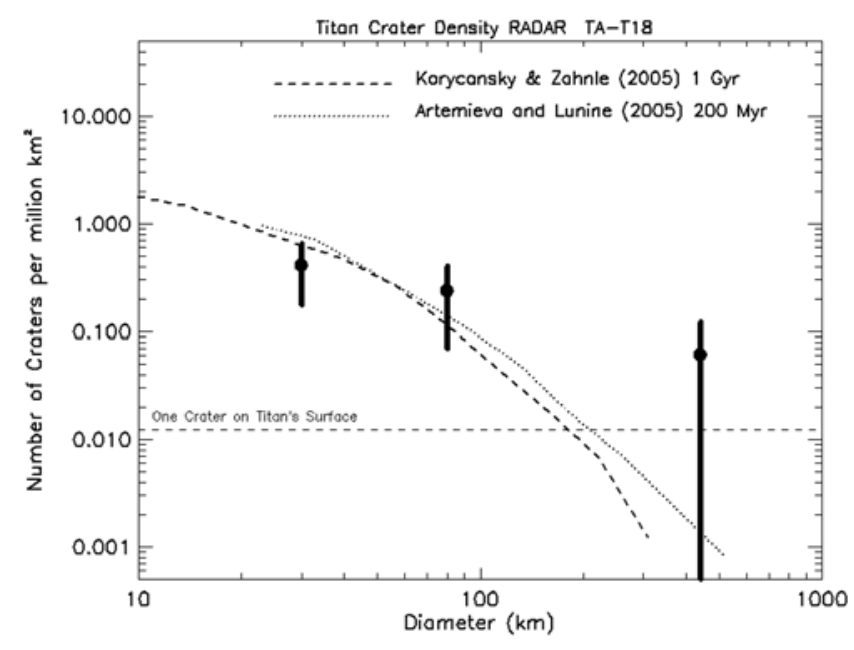

Figure 5. Observed cumulative size-frequency distribution compared with two models of the crater population that incorporate projectile disruption by the atmosphere, and hence a lower slope at small crater sizes. The observed crater density slope is shallower than the models for $\sim 80 \mathrm{~km}$ craters, suggesting a surface obliteration process (burial, erosion). Lower crater retention ages (discrepant by a factor of $\sim 5$ between the two models) are indicated for the smaller craters than for the $440 \mathrm{~km}$ Menrva, for which a $\sim \mathrm{Gyr}$ retention age is suggested by both.

present in so-far unimaged areas. For modest craters such as the $80 \mathrm{~km}$ crater Sinlap, the probability is $\sim 15 \%$, only marginally larger than the percentage area covered so far. For very large craters, e.g., $1000 \mathrm{~km}$ diameter, the nondetection indicates a $55 \%$ probability that no such crater exists anywhere on Titan.

\section{Crater Density and Interpretation}

[14] The observed cumulative crater density is shown in Figure 4, for the three named craters, plus a version of the curve that incorporates a representative number of additional likely features to illustrate that in fact the uncertain number of such structures does not affect our conclusions. The number of craters is sufficiently small that a differential crater distribution (craters per $2^{\wedge} 0.5$ size bin, or 'Hartmann plot') and normalized cumulative distribution ('R-plot') are not yet meaningful, thus we show only cumulative plots. It can be seen that the detection so far of a $\sim 400 \mathrm{~km}$ crater is consistent with the overall (old) density of such craters on other Saturnian satellites. However, the number of $20-$ $100 \mathrm{~km}$ craters, even if augmented by plausible but not certain impact structures, is far lower - by a factor of $30-$ 300 , indicating a much younger surface.

[15] The density of craters is $\sim 5$ times higher than Earth in terms of number per unit area. However, even ignoring the fact that the impactor population at Saturn is likely different from that affecting the terrestrial planets, a casual comparison is nonetheless worthwhile. The density does not mean Titan's surface is $5 \times$ older, since resurfacing on Titan may be expected on purely energetic grounds to be less vigorous [Lorenz and Lunine, 2005] - the heat flux driving erosion is a factor of $\sim 100-1000$ lower on Titan, and the heat flux driving convection and tectonics on Titan is a factor of $\sim 10$ lower than on Earth. Taking these factors into account, Lorenz and Lunine [2005] predicted a more 'balanced' mix of processes for Titan than Earth (i.e., while erosion and endogenic processes are dominant on both Titan and Earth over cratering, the factor is less on Titan), thus a larger fraction of Titan's surface shows impact features than the Earth. For reference, applying Hartmann's [2005] recent isochrons to the terrestrial crater population yields an age that depends on the size of crater considered, but gives a crater retention age of Earth's continents for 20-100 km diameter craters of $100 \mathrm{Myr}-1 \mathrm{Gyr}$.

[16] We must apply models of the Saturnian impactor population that take into account the shielding effect of the atmosphere to derive an age for Titan's surface - see Figure 5. Note that this is simply a 'crater retention age'as mentioned above, this is size-dependent, and only reflects the ability of the surface to retain observable impact crater morphology, rather than any implication about the last rainfall or dune movement. The age for Menrva is rather high (order a few Gyr) and highly uncertain. However, the age of 20-100 km craters, applying the Korycansky and Zahnle [2005] model, is remarkably only a Gyr at most. A different model (although itself derived from previous work by Zahnle) by Artemieva and Lunine [2005] suggests a slightly younger age, of the order of 100-500 Myr. Neither model considers the added impactor population from a putative breakup of the precursor to Hyperion [Farinella et al., 1997], although if this happened early in Titan's history and the surface is as youthful as derived here, the impact signature of that breakup would likely be obliterated. It may be noted that models of Titan's crustal evolution show it thickening substantially $\sim 500$ Myr ago.

[17] A more detailed investigation of the model assumptions in each case is unlikely to be very informative, in that absolute crater ages are controversial and the statistical precision of the Titan results is not yet high. The qualitative conclusion, that Titan's surface is on average rather youngcomparable with the Earth or Venus - is nonetheless robust, and agrees with the estimate of a few 100 Myr based on larger area coverage but less secure crater identifications in the work of Porco et al. [2005]. Why Titan should be so young is not yet certain since the few craters at hand do not permit much generalization: the preferential preservation of rims such as Guabonito would be consistent with burial or viscous relation, yet the similar-sized Sinlap shows no such rim, and retains a sharp floor $1300 \mathrm{~m}$ deep [Elachi et al., 2006]. It is clear that larger craters are better preserved (i.e., are older) - perhaps arguing that viscous relaxation, which destroys long-wavelength topography fastest, is not the dominant process of obliteration, leaving erosion and burial as the principal processes. The pronounced east-west difference in the preservation state of Menrva, and the evident prograde trend of dunes at Sinlap, Guabonito and Ksa, are also consistent with these atmospheric processes being responsible for crater destruction. Although cryovolcanism doubtless plays a role in resurfacing Titan, we have not yet seen examples of craters partially destroyed by such processes.

[18] It may be noted that except for feature $\mathrm{F}$, all the discussed impact structures are at longitudes of $0-180^{\circ} \mathrm{W}$, i.e., on Titan's leading hemisphere. The impactor model of 
Korycansky and Zahnle [2005] indeed suggests that the leading hemisphere should have a density of about 5 times higher than the trailing side. If this apparent agreement is not merely fortuitous, one interpretation is that Titan has been a synchronous rotator throughout most of its (observable) history. We must acknowledge, however, that most of our coverage is over the leading hemisphere-better statistics will emerge as the coverage builds up throughout the mission. It is also evident that the mountains of Xanadu (T13, leading) and the dune fields of Belet (T8, trailing) are rather distinct terrains, and so resurfacing processes may have a longitudinal dependence that can distort the observed leading-trailing asymmetry in remnant crater density. Future observations of the leading hemisphere will be needed to determine whether the asymmetry is real, and whether due to inhomogeneous resurfacing rather than inhomogeneous cratering density.

[19] A final remark may be made about the paucity of small craters. In the present atmosphere, many projectiles that form craters of less than about $20 \mathrm{~km}$ diameter are disrupted by the atmosphere, and thus the differential distribution at these sizes is expected to be small. (But not zero - some small fraction of iron impactors and large fragments will yield some small craters [see Korycansky and Zahnle, 2005]. The fact that very few, if any, $<20 \mathrm{~km}$ craters are observed implies that Titan's atmosphere has not been thinner by a factor of several ('collapsed') in the recent past - although the low age means that this is recent indeed, and thus not constraining over the multi-Gyr periods of Titan's history when such large-scale atmospheric changes may be expected. [Tobie et al., 2006]. As Figure 2 shows, however, structures of only a few $\mathrm{km}$ diameter are difficult to identify robustly in our data with $300-1000 \mathrm{~m}$ resolution.

\section{Conclusions}

[20] A survey of Titan's crater population observed with SAR imaging on Cassini's Titan flybys through T18 (August 2006) indicates only 3-10 impact structures, instead of the hundreds that would be expected. The size distribution is even flatter than would be expected from atmospheric shielding, suggesting additional obliteration processes. Initial investigation suggests that erosion and burial are the principal processes involved, as on Earth, yielding a very youthful surface indicative of active geological processes.
[21] Acknowledgments. The authors gratefully acknowledge those who designed, developed, and operate the Cassini/Huygens mission. The Cassini/Huygens Project is a joint endeavor of the National Aeronautics and Space Administration (NASA), the European Space Agency (ESA), and the Italian Space Agency (ASI) and is managed by the Jet Propulsion Laboratory, California Institute of Technology, under a contract with NASA. The authors thank members of the Cassini RADAR Team for their assistance. We also thank $\mathrm{Ph}$. Paillou and an anonymous referee for useful comments.

\section{References}

Artemieva, N., and J. I. Lunine (2005), Impact cratering on Titan II, Global melt, escaping ejecta, and aquueous alteration of surface organics, Icarus, $175,522-533$.

Elachi, C., et al. (2005), Cassini Radar views the surface of titan, Science, $308,970-974$.

Elachi, C., et al. (2006), Titan Radar mapper observations from Cassini's TA and T3 flybys, Nature, 441, 709-713.

Engel, S., J. I. Lunine, and W. K. Hartmann (1995), Cratering on Titan and implications for Titan's atmospheric history, Planet. Space Sci., 43, 131154.

Farinella, P., F. Marzari, and S. Matteoli (1997), The disruption of Hyperion and the origin of Titan's atmosphere, Astron. J., 113, 2312-2316.

Hartmann, W. K. (2005), Martian cratering 8: Isochron refinement and the chronology of Mars, Icarus, 174, 294-320.

Ivanov, B. A., A. T. Basilevsky, and G. Neukum (1997), Atmospheric entry of large meteoroids: Implication to Titan, Planet. Space Sci., 45, $993-$ 1007 .

Korycansky, D. G., and K. J. Zahnle (2005), Modeling crater populations on Venus and Titan, Planet. Space Sci., 52, 695-710.

Lorenz, R. D., and J. I. Lunine (2005), Titan's surface before Cassini, Planet. Space Sci., 53, 557-576.

Lorenz, R. D. (1997), Impacts and cratering on Titan - a pre-Cassini view, Planet. Space Sci., 45, 1009-1019.

Phillips, R. J., R. E. Arvidson, J. M. Boyce, D. B. Campbell, J. E. Guest, G. G. Schaber, and L. A. Soderblom (1991), Impact craters on Venus: Initial results from Magellan, Science, 252, 288-297.

Porco, C. C., et al. (2005), Imaging of Titan from the Cassini spacecraft, Nature, 434, 159-168.

Stofan, E. R., et al. (2007), The lakes of Titan, Nature, 445, 61-64.

Tobie, G., J. I. Lunine, and C. Sotin (2006), Episodic outgassing as the origin of atmospheric methane on Titan, Nature, 440, 61-64.

Y. Z. Anderson, R. M. Lopes, K. L. Mitchell, F. Paganelli, and S. D. Wall, Jet Propulsion Laboratory, California Institute of Technology, Pasadena, CA 91109, USA.

R. D. Lorenz, Space Department, Applied Physics Laboratory, Johns Hopkins University, Laurel, MD 20723, USA. (ralph.lorenz@jhuapl.edu)

J. I. Lunine, Lunar and Planetary Laboratory, University of Arizona, Tucson, AZ 85721, USA.

E. R. Stofan, Proxemy Research, 20528 Farcroft Lane, Bowie, MD 20715, USA.

C. Tsai and L. Wye, Department of Electrical Engineering, Stanford University, Stanford, CA 94305, USA.

C. A. Wood, Center for Education Technology, Wheeling Jesuit College, Wheeling, WV 26003, USA.

H. Zebker, Department of Geophysics, Stanford University, Stanford, CA 94305, USA. 